\title{
Effects of Andrographis paniculata (Burm. F.) Extract on Diabetic Nephropathy in Rats
}

\author{
Rachmat Hidayat*1 and Patricia Wulandari ${ }^{2}$
}

\begin{abstract}
Background: Hyperglycemia and accumulation of advanced glycation end products (AGEs) play a significant role in the development of diabetic nephropathy. Andrographis paniculata (AP) is a plant with high flavonoid content with the potential to suppress oxidative stress activity in cells and tissue. This study was aimed to investigate the role of Andrographis paniculata extract (APE) in protecting kidney damage due to the formation of AGEs in the renal glomerulus in diabetic rats.

Methods: A total of 30 male Sprague Dawley rats were randomly divided into five groups as follows: normal control group, streptozocin (STZ) induced diabetic group, STZ-induced diabetic group with AP extract (100 mg/kg BW), STZ-induced diabetic rats with AP extract (200 mg/kg BW), and STZinduced diabetic rats with APE (400 mg/ kg BW). Blood glucose levels were measured before treatment and after treatment. Serum and urine parameters were determined. Antioxidant enzymes and lipid peroxide levels were determined in the kidney along with histopathological examination.

Results: The finding of this study showed that treatment APE at the dose of $200 \mathrm{mg} / \mathrm{kg}$ and $400 \mathrm{mg} / \mathrm{kg}$ ameliorated kidney hypertrophy index. SOD, catalase, and GSH activities significantly decreased in the kidney of STZ-diabetic rats compared to the normal control rats. Treatment with APE significantly decreased malondialdehyde level at the dose of 200 and $400 \mathrm{mg} / \mathrm{kg} \mathrm{BW}$.

Conclusions: This study revealed evidence for improving diabetic retinopathy in male rats treated with Andrographis paniculata extract. APE significantly decreased oxidative stress activities in kidney of diabetic rats.
\end{abstract}

Keywords: Andrographis, Diabetic Nephropathies, Streptozocin, Rats, Oxidative Stress.

\section{Introduction}

Diabetes mellitus is a metabolic disease indicated by hyperglycemia resulting from defects in insulin secretion, insulin action, or both. Sustained hyperglycemia is further associated with long-term damage, dysfunction, and failure of various organs and is a major factor in developing many complications in patients with diabetes $(1,2)$. Moreover, diabetes is the most frequent cause of progressive kidney failure leading to dialysis or transplantation (3). Nephropathy is reported to occur in $30-40 \%$ of patients with diabetes and has become a leading cause of end-stage renal failure in the world $(4,5)$. Diabetic nephropathy is characterized by structural and functional abnormalities in the kidney (6). Uncontrolled blood glucose and accumulation of advanced glycation end products (AGEs) play a significant role in the development of diabetic nephropathy (7).

Moreover, advanced glycation end products have been implicated in tissue damage related to diabetic nephropathy. The clinical symptoms and pathological hallmarks of diabetic nephropathy include urinary albumin excretion and accumulation of extracellular matrix, thickening of basement membranes, mesangial expansion, hypertrophy, and glomerular epithelial cell (podocyte) loss within the 
glomeruli (8). Patients with diabetic nephropathy have a continuous decline in glomerular function. Antihypertensive agents, particularly those targeting the reninangiotensin system, are reported to be the most effective treatments for progressive diabetic nephropathy to date (9). However, these treatments are not able to prevent the onset of diabetic nephropathy.

Plants have been used as traditional medicine sources for years, and many studies had identified plants as good sources of new treatments for many life-threatening diseases. Plants with antidiabetic potential are believed to be valuable sources for new oral hypoglycemic agents. Andrographis paniculata (Burm. F.) have been demonstrated as potential sources of antidiabetic properties $(10,11)$. Andrographis paniculata (AP) originated from India is widely grown in Southeast Asian countries, including Indonesia, and often used as jamu pahitan (Indonesian traditional medicine drink) to maintain health and treat many diseases such as tonsillitis, chancre, typhoid fever, diabetes, eczema, the common cold, diphtheria, depurative, epilepsy, gonorrhoea, syphilis, and dandruff. The extract of AP (APE) was demonstrated to reduce blood glucose levels in diabetic rats in several studies $(12,13)$. An in vitro study of the extract discovered a strong insulin-secreting effect in BRIN-BD11 cells, a pancreatic cell line expressing insulin, and glucokinase (14). The main active component in APE is andrographolide, and it produced a hypoglycemic effect by elevating the mRNA and protein levels of GLUT4 in rats with diabetes mellitus (10).

This plant is a kind of shrub with high flavonoid content, where flavonoids are very potent antioxidants. Antioxidants are compounds with the potential to suppress oxidative stress activity in cells where the accumulation of AGEs in cells and tissues plays a role in the initiation of oxidative stress in cells and tissues. Oxidative stress inhibition inhibits the inflammatory cascade and inhibition of cell and tissue death and damage. This study is the first research that aimed to specifically explore the role of AP extract in protecting kidney damage due to the formation of AGEs in the renal glomerulus in diabetes mellitus.

\section{Materials and Methods Authentication of plant}

Andrographis paniculata (AP) leaves were collected from Palembang, South Sumatra, Indonesia, in the month of January 2020 and identified by Botany Department, Faculty of Sciences, Universitas Sriwijaya, Palembang, Indonesia. A voucher specimen (No. 2020/15) was deposited at the Department of Biology, Faculty of Medicine, Universitas Sriwijaya, Palembang, Indonesia.

\section{APE preparation}

Andrographis paniculata (AP) simplicia were prepared to study their hypoglycemic effects and investigate the plant's basic phytochemical composition. The water extract (WE), similar to brew traditional tea, was made by boiling 20 $\mathrm{g}$ of the dry plant material in water $(500 \mathrm{~mL})$, followed by filtration and lyophilization. The ethanol-water extract (EWE) was prepared by adding $20 \mathrm{~g}$ of the plant material to $800 \mathrm{ml}$ of a mixture of ethanol and water (50:50). The extract was then heated at $40{ }^{\circ} \mathrm{C}$ for four hours; thereafter, it was filtered three times, followed by evaporation in a Büchi rotary evaporator. Phytochemical test of the plant was performed by extracting $60 \mathrm{~g}$ using a Soxhlet extractor with n-hexane (HE) followed by methanol. The dry methanol extract was partitioned with butanol and water at a 1:1 ratio, and the butanolic phase was then dried (BE), followed by evaporation of all of the extracts in a rotary Büchi evaporator. All of the extracts were kept at $-4{ }^{\circ} \mathrm{C}$ until use.

\section{Thin-layer chromatography (TLC) analysis}

The extracts were analyzed using the standard TLC method. In summary, the sample was applied to the Merck Plates $10 \mathrm{~mm} \times 10 \mathrm{~mm}$ 60 F254 containing a solvent system. For alkaloids, a mixture containing dichloromethane, 85: $\mathrm{MeOH}$, 14: $\mathrm{NH} 4 \mathrm{OH}$ (25\%), 1, was used, and the plates were visualized using Dragendorff's Reagent. For flavonoids, a mixture containing ethyl acetate, 
60: formic acid, 10: acetic acid, 10: water, 20 is used. Both plates were visualized using diphenylborinic acid. For terpenes, $20 \mathrm{ml}$ of the mixture containing ethyl acetate, 6 : HE 14, is used. The plate was visualized using vanillin (15).

\section{Animals \\ Healthy male Sprague Dawley rats weighing between 250 and $300 \mathrm{~g}$ were procured from Eureka Laboratory and Experimental Animal Breeding Co., Ltd (Palembang, Indonesia) and maintained in polypropylene cages at the ambient temperature of $22 \pm 1{ }^{\circ} \mathrm{C}$ and relative humidity of $50-60 \%$ with a $12 \mathrm{~h}$ light/dark cycle in the registered animal house (87/2020/ Biotek) at Faculty of Medicine, Universitas Sriwijaya, Palembang, Indonesia. Throughout the experimental period, the animals were fed with standard pellet diet (Comfeed, Indonesia) and water ad libitum.}

\section{Experimental procedure}

A single injection of streptozotocin-induced diabetes (STZ, $45 \mathrm{mg} / \mathrm{kg}$, i.p. freshly prepared in $0.1 \mathrm{M}$ citrate buffer $\mathrm{pH} 4.5$ ) in rats (16). The control group received an equal volume of vehicle (0.1 M citrate buffer, $\mathrm{pH} 4.5)$. Blood glucose level was measured from the tail vein using a glucose meter (ACCU-CHEK advantage, Hangzhou, China) seven days after induction. Rats with blood glucose levels above $14 \mathrm{mmol} / \mathrm{L}$ were considered diabetic and were used for further study by initiating the treatment. Andrographis paniculata extract (APE) was administered daily to rats per oral for eight weeks. The animals were divided into 5 groups: Normal control group $(n=6), S T Z$ induced diabetic group $(n=6)$, STZ-induced diabetic group treated with APE $100 \mathrm{mg} / \mathrm{kg}$ $(n=6)$, STZ-induced diabetic group treated with APE $200 \mathrm{mg} / \mathrm{kg}(\mathrm{n}=6)$ and STZ-induced diabetic group treated with APE $400 \mathrm{mg} / \mathrm{kg}$ $(n=6)$. The three doses of the extract were selected based on the acute oral toxicity study reported on the plant and the previous studies carried out on the plant. Blood glucose levels were measured before treatment and on 1st, 2nd, 4th, 6th \& 8th weeks. The bodyweight of each animal was determined at the initiation and end of the study. On the completion of 8 weeks, blood was withdrawn via retro-orbital plexus. Blood samples were centrifuged at 1300x $\mathrm{g}$ for separation of serum and stored at $20{ }^{\circ} \mathrm{C}$ until assay. At the end of the experimental period, animals were perfused with saline to remove the organs' traces of blood cells.

\section{Preparation renal homogenate}

Before sacrifice, the rats were anaesthetized with chloroform inhalation. Immediately after sacrifice, both the kidneys were dissected, rinsed with isotonic saline, and weighed. After weighing, each kidney was cut into two halves. One half was used for histopathological evaluation. The other half was minced, and a homogenate was prepared with $10 \%(\mathrm{w} / \mathrm{v})$ phosphate-buffered (0.1 M, pH 7.4) using a homogenizer. The kidney homogenate was centrifuged, and the supernatant was estimated for kidney antioxidant parameters.

\section{Serum and urine parameters}

Serum was used to estimate albumin, creatinine, blood urea nitrogen (BUN), cholesterol and triglyceride levels. Pooled $24 \mathrm{~h}$ urine was evaluated for creatinine and albumin value. The estimation of the above-mentioned parameters was carried out using biochemical kits (ACCUREX, Biomedical Pvt. Ltd). AGEs level was examined in kidney supernatant. It was measured by ELISA, according to the manufacturer's protocols. Briefly, $50 \mu \mathrm{l}$ of standard diluent or serum samples were added to the well and incubated at $37{ }^{\circ} \mathrm{C}$ for 30 minutes. After the plates were washed, $100 \mu 1$ of the biotinylated antibody solution was added and set for 30 minutes at $37^{\circ} \mathrm{C}$. After washing three times, $50 \mathrm{ul}$ avidin-peroxidase complex solution was added and incubated for 15 minutes at $37^{\circ} \mathrm{C}$. After washing, $50 \mu \mathrm{l}$ of tetramethylbenzidine color solution was added and set in the dark for 15 minutes at $37{ }^{\circ} \mathrm{C}$. Finally, a 50 ul stop solution was added to stop the reaction. The absorbance was measured at $450 \mathrm{~nm}$ using an ELISA reader (Epoch, Biotek, Winooski, VT, United States). 


\section{Kidney antioxidant parameters}

5-5-dithiobis (2-nitrobenzoic acid) (DTNB) reagent was used to estimate reduced glutathione (GSH) level in tissue homogenates, and the absorbance was read at $412 \mathrm{~nm}$. The amount of GSH in the sample was calculated in microgram per $\mathrm{ml}$ from a standard curve obtained and represented in GSH per total tissue protein. Evaluation of kidney homogenate for lipid peroxidation levels, superoxide dismutase (SOD), and catalase (CAT) activities were carried out following the method published by Nishi and Kumar (17) and Halliwell and Chirico (18).

\section{Statistical analysis}

Data were analyzed using SPSS 25.0 (SPSS, Inc., Armonk, NY, United States) by one-way analysis of variance (ANOVA) followed by post hoc analysis was carried out to assess the difference in mean expression levels of each parameter. Figures were generated using Adobe Photoshop (Adobe Software, San Diego, CA, United States). All data were expressed mean + standard deviation (SD), and a p-value of 0.05 was considered statistically significant.

\section{Ethical approval}

All experiments in this study complied with the bioethical research established by the Institutional Animal Care and Use Committee, Faculty of Medicine of Universitas Sriwijaya (No. 278/kptfkunsrirsmh/2020); and its methodology conforms to the published guide "Principle of laboratory animal care" (19).

\section{Results}

During the 8-week experiment, STZ induced diabetic rats exhibited significant weight loss when compared with normal rats. At the end of 8 weeks of treatment, the bodyweight of rats treated with APE at the dose of $200 \mathrm{mg} / \mathrm{kg}$ and $400 \mathrm{mg} / \mathrm{kg}$ was significantly increased than the diabetic control group (Table 1). The kidney weight and kidney hypertrophy index in STZ induced diabetic rats were significantly increased as compared to those in the normal control group. Treatment of STZ induced diabetic rats with APE at the dose of $200 \mathrm{mg} / \mathrm{kg}$ and $400 \mathrm{mg} / \mathrm{kg}$ ameliorated kidney hypertrophy index (Table 1).

Table 1. Comparison of body weight and kidney weight between treatment groups.

\begin{tabular}{llll}
\hline GROUP & $\begin{array}{l}\text { BODY WEIGHT } \\
\text { (GRAM) AT 8 WEEK } \\
\text { MEAN } \pm \text { SEM }\end{array}$ & $\begin{array}{l}\text { KIDNEY WEIGHT } \\
\text { (GRAM) } \\
\text { MEAN } \pm \text { SEM }\end{array}$ & $\begin{array}{l}\text { KIDNEY } \\
\text { HYPERTROPHY } \\
\text { (KIDNEY } \\
\text { WEIGHT/BODY } \\
\text { WEIGHT) \% } \\
\text { MEAN } \pm \text { SEM }\end{array}$ \\
\hline CONTROL & $404.17 \pm 9.82$ & $1.17 \pm 0.04$ & $0.3 \pm 0.01$ \\
DIABETICS & $218.73 \pm 15.63 \#$ & $1.73 \pm 0.1 \#$ & $0.63 \pm 0.03 \#$ \\
$\mathbf{1 0 0}$ MG/KGBB APE & $233.33 \pm 10.96$ & $1.23 \pm 0.04$ & $0.53 \pm 0.03$ \\
$\mathbf{2 0 0}$ MG/KGBB APE & $266.17 \pm 10.02^{*}$ & $1.17 \pm 0.04 *$ & $0.43 \pm 0.02^{*}$ \\
$\mathbf{4 0 0}$ MG/KGBB APE & $318.67 \pm 10.72^{* *}$ & $1.11 \pm 0.03^{* *}$ & $0.37 \pm 0.06^{* *}$ \\
\hline
\end{tabular}

*Treatment group was compared with diabetic control, $\mathrm{p}<0.05$; ** Treatment group was compared with diabetic control, $\mathrm{p}<$ 0.05; \# Diabetic group was compared with normal control, $\mathrm{p}<0.05$, using one-way ANOVA with Pos hoc test.

Administration of STZ led to a significant increase in fasting blood glucose levels in the diabetic group compared to the normal control group throughout eight weeks after induction (Fig. 1). Furthermore, STZ-diabetic rats treated with $100 \mathrm{mg} / \mathrm{kg}$ APE demonstrated a decrease in the fasting blood glucose levels at the end of 8 weeks, whereas STZ-diabetic rats treated with $200 \mathrm{mg} / \mathrm{kg}$ and $400 \mathrm{mg} / \mathrm{kg}$ APE showed a significant decrease in the fasting blood glucose levels at the end of 6 weeks (Fig. 1). 


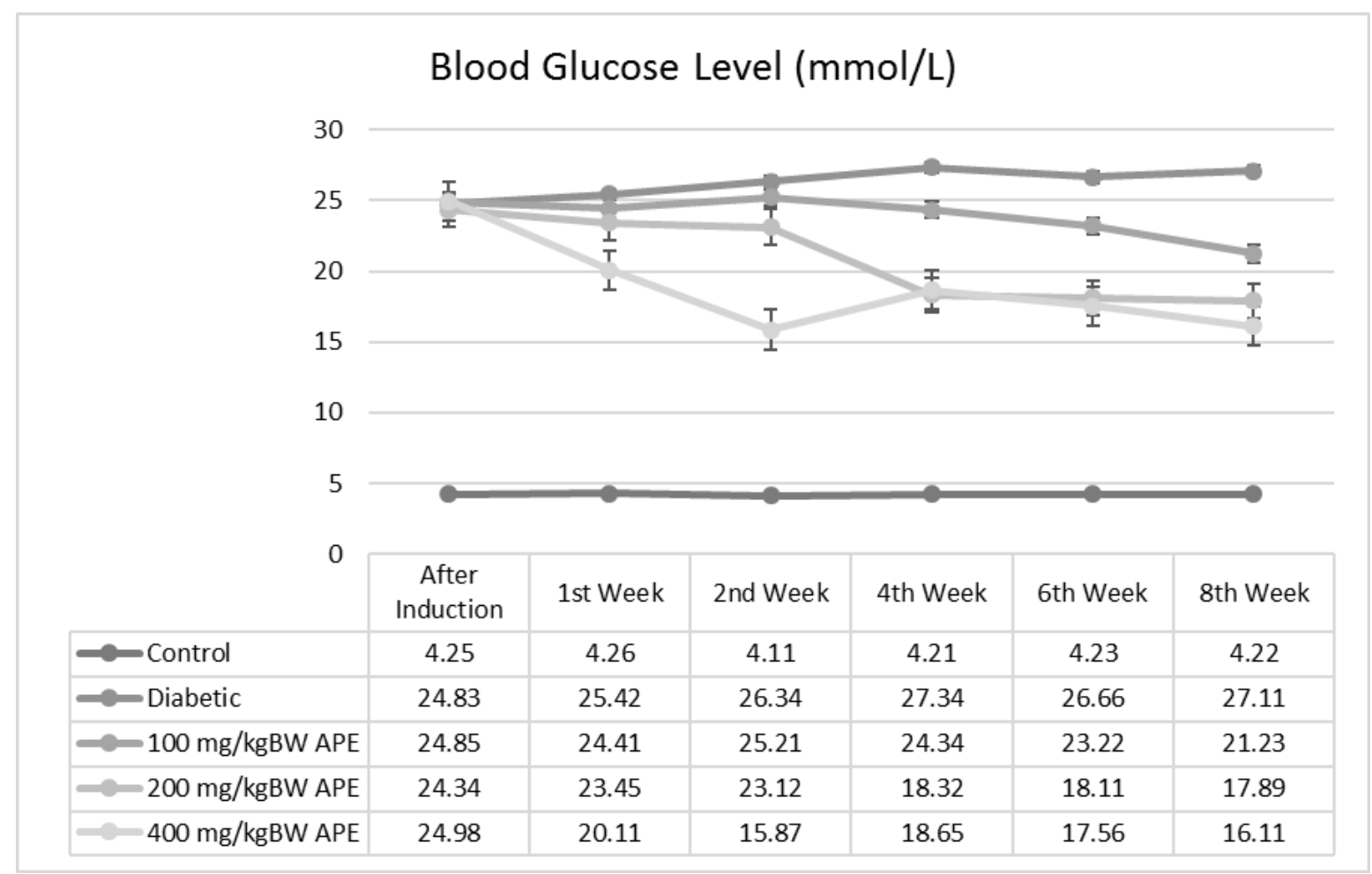

Fig. 1. Comparison of blood sugar levels between groups. A significant increase is shown in blood glucose level of diabetic group. A gradual decrease of blood glucose level was occurred after first week of APE $200 \mathrm{mg} / \mathrm{kg}$ BW administration. Notes: APE: Andrographis paniculata extract.

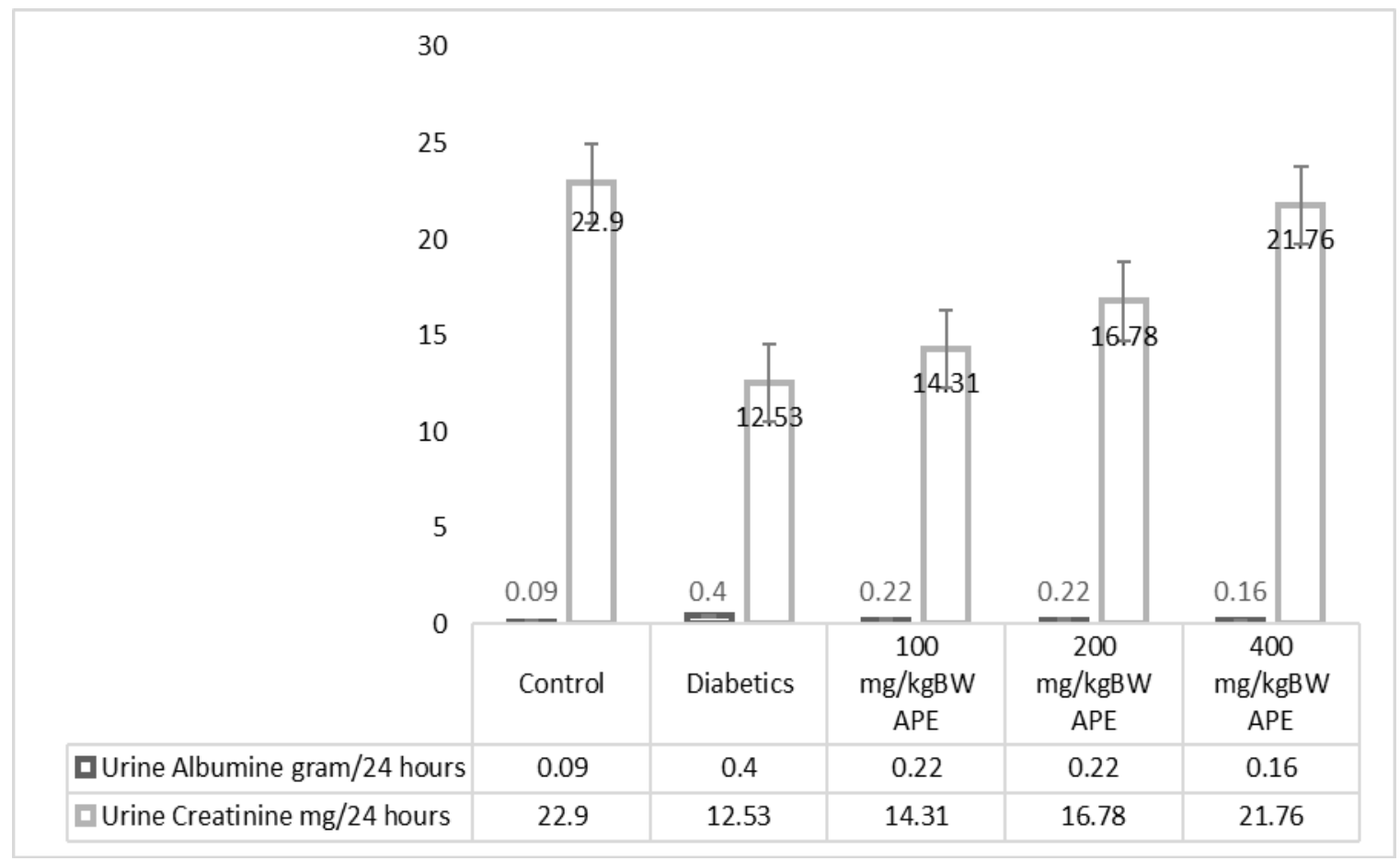

Fig. 2. Comparison of urine albumin and urine creatinine levels between groups. Low urine albumin level and increased urine creatine level are marked in highest dose of $A$. paniculata extract $(400 \mathrm{mg} / \mathrm{kgBW})$. 


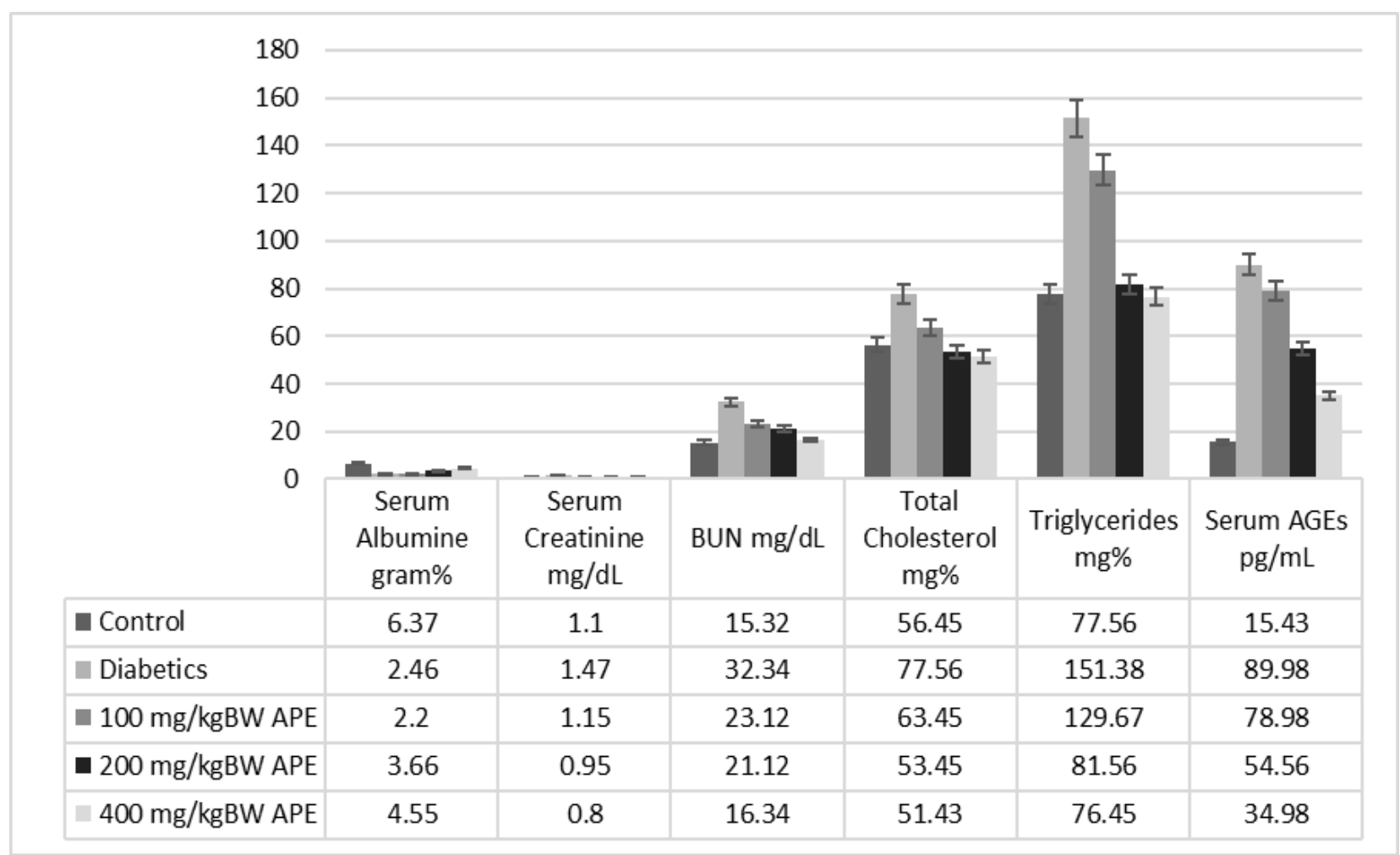

Fig. 3. Comparison of serum albumin, serum creatinine, blood urea nitrogen (BUN), total cholesterol, triglycerides, serum AGEs between groups. Level of blood urea nitrogen, total cholesterol, triglycerides and AGEs serum were decreased in line with A. paniculata dose.

The STZ-induced diabetic rats showed a significant decrease in creatinine level in urine with a significant increase in serum compared to the normal control group (Fig. 2). After treatment with APE, the creatinine content increased significantly in urine with a significant decrease in serum. The STZdiabetic rats showed a significant increase in the urine's albumin content, with a significant decrease in serum level compared to the normal group. The albumin level of the STZdiabetic rats treated with APE was brought back to normal. The STZ-diabetic rats exhibited a significant increase in the blood urea nitrogen content, whereas treatment with MPGL significantly decreased this level. In STZ-induced diabetic rats, a significant increase in serum triglycerides and cholesterol was observed compared to the normal control group. However, STZ-diabetic rats treated with APE demonstrated a significant decrease in the level of triglycerides and cholesterol. Advanced glycation end products were significantly higher in the STZ- diabetic rats when compared with normal control rats. Administration of APE to STZ-diabetic rats reduced the advanced glycation end products significantly compared to the negative control group (Figs. 2 \& 3).

Significantly higher levels of malondialdehyde (MDA) (a marker of lipid peroxidation) were detected in the kidney of STZ-diabetic rats. SOD, Catalase and GSH activities were significantly decreased in the kidney of STZ-diabetic rats as compared to the normal control rats. Treatment with APE significantly decreased the increased level of MDA at the dose of 200 and $400 \mathrm{mg} / \mathrm{kg}$ and significantly increased the decreased activities of SOD and GSH at all dose levels. Significant elevation of catalase activity was observed at the dose of $400 \mathrm{mg} / \mathrm{kg}$ of APE (Fig. 4).

Phytochemical test of Andrographis paniculata extract shows that each extract is rich in flavonoids. Flavonoids are the main secondary metabolites and believed to play a role in inhibiting inflammation and oxidants due to the accumulation of AGEs (20). 


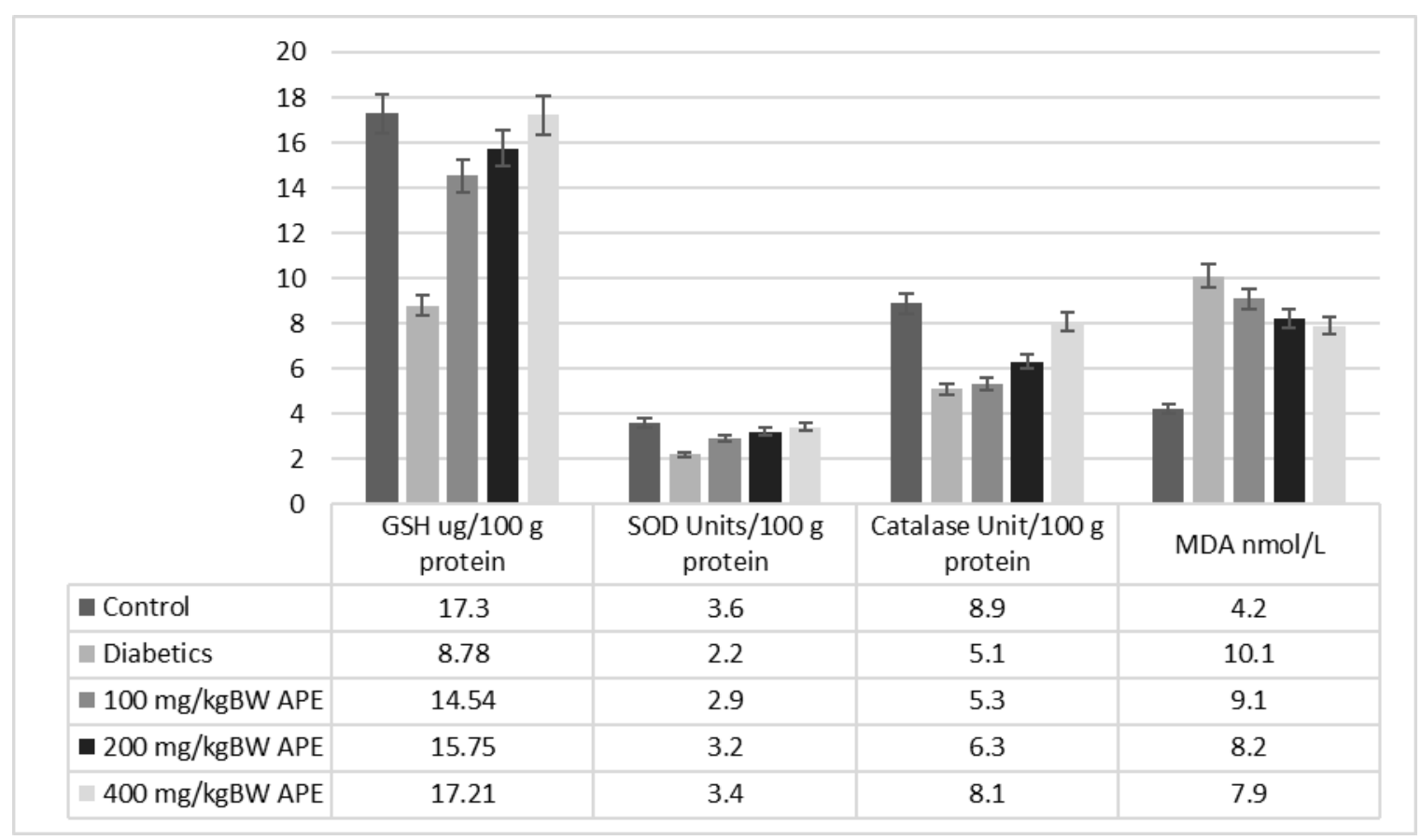

Fig. 4. Comparison of GSH, SOD, Catalase and MDA levels between groups.

\section{Discussion}

Diabetes is a complex endocrine disorder involving insulin deficiency which leads to chronic hyperglycemia with disturbances of carbohydrate, fat and protein metabolism. It is estimated that more than 300 million people worldwide will have diabetes mellitus by 2025 (21). Diabetic nephropathy, a serious complication of diabetes mellitus, is the most common cause of end stage renal failure. About $15-25 \%$ of type 1 diabetes patients and $30-40 \%$ of patients with type 2 diabetes suffer from diabetic nephropathy (22). In spite of the availability of therapeutic agents which retard the progression of diabetic nephropathy, there has been renewed interest in the use of herbal medicines in order to prevent the genesis of this complication. Andrographis paniculata L., a deciduous shrub, the leaves of which are rich in polyphenolic compounds including tannins and flavonoids (12) has aroused great interest due to its antidiabetic potential which could be considered as a lead to further study the effect of this part of the plant on diabetic complications such as nephropathy. Taking this into consideration, in our present investigation, we have evaluated the protective effects of APE on STZ-induced diabetic nephropathy in rats. Streptozotocin has been an agent of choice to induce experimental diabetes mellitus due to its ability to induce specific necrosis of the pancreatic beta cells resulting in degranulation and loss of capacity to secrete insulin $(23,24)$. Thus, in the present study, STZ was used for induction of diabetes in rats. Administration of STZ led to significant increase in blood glucose level which was lowered on treatment with APE thus confirming the antihyperglycemic activity of the extract as reported by earlier established studies (23). However, up to 4 weeks no significant difference was observed in the glucose level of APE treated groups as compared to diabetic group. One of the possible reasons for this may be that the extract does not possess a strong anti-diabetic activity. The concentration of active principles at the given doses might not be capable in controlling hyperglycemia in the initial weeks. The active principles in the extract might take time to reach sufficient concentration at the target site. It could also be due to principles such as reducing sugars or other carbohydrates which might interfere with the onset of hypoglycemic effect of APE. Several reports have stated the diminished hypoglycemic responses of extract due to the presence of reducing sugars which could give rise to free glucose after digestion that may tend to rise blood glucose levels in the 
face of the hypoglycemic actions by the active hypoglycemic agents $(25,26)$. STZ-induced diabetes is associated with significant reduction in the body weight due to hyperglycemia, hypoinsulinemia, increased muscle wasting and loss of tissue proteins (27). The body weight of STZ-diabetic rats was progressively reduced and treatment with APE improved the body weight significantly, thus indicating prevention of muscle tissue damage caused due to hyperglycemia.

Increase in the weight of kidney (hypertrophy) in proportion to the body weight is observed in STZ-induced rats. Local alterations in the production of one or more growth factors such as over expression of transforming growth factor-beta 1 in the kidney especially in proximal convoluted tubules cells and glomerular mesangial cells are proposed in the development of renal hypertrophy. An increase in the rate of protein synthesis and/or decrease in the degradation of renal extracellular components might also lead to renal hypertrophy (27). Treatment reduced kidney/body weight ratio, thus demonstrating reversal of kidney hypertrophy in STZ-diabetic rats. Diabetes mellitus leads to fatty liver, hypercholesterolemia and hypertriglyceridemia. Moreover, elevated cholesterol levels are associated with diabetic nephropathy (28). These increased levels were reversed towards normal after treatment with APE, thus demonstrating its potential to improve lipid metabolism. Hypoalbuminaemia is considered as a strongest predictor of death in patients with renal failure. Albumin is by far the most abundant protein in nephrotic urine. In STZ-diabetic rats, serum albumin concentration was decreased significantly with an increase in the albumin levels in urine, thus demonstrating that albuminuria was related to deteriorating kidney function (16). Treatment with APE normalized these levels thus, exhibiting its beneficial role against microalbuminuria. Increased serum creatinine level and BUN along with decreased excretion of creatinine in the urine are indicators of the development of diabetic nephropathy (28). Reversal of these effects was observed in STZ-diabetic rats treated with APE. The formation of AGEs in renal tissue plays a crucial role in the development of diabetic nephropathy. The irreversible formation of AGEs affects proteins and lipids thus causing damage to the blood vessels and kidneys. AGEs are found in almost all tissues examined from STZ-induced diabetic rats. Moreover, kidneys are more susceptible to AGE formation than other tissues. Increased level of AGE was found in the serum of STZdiabetic rats whereas treatment with APE lowered the elevated AGE levels (29). Thus APE exhibited the potential to protect the kidney by decreasing the formation of AGE in the circulation of the STZ-diabetic rats.

Hyperglycemia leads to increased production of reactive oxygen species (ROS) which are involved in the etiology of several diabetic complications including diabetic nephropathy (30). The reactive oxygen species deplete the antioxidant defenses of the cell thus making it more susceptible to oxidative damage (31). It further targets lipid, DNA and protein leading to their oxidation which further leads to changes in cellular structure and function (32). GSH plays a crucial role as a free radical scavenger in addition to the maintenance of plasma antioxidant status. Superoxide dismutase converts superoxide to a less reactive ROS, hydrogen peroxide which is further reduced to water by catalase. Thus, catalase assists SOD in the complete neutralization of ROS (30,32). Malondialdehyde (MDA), a latestage lipid oxidation by product, is an important indicator of free radical-induced lipid peroxidation (29). Increased MDA levels were suppressed in STZ-diabetic rats treated with APE. In addition to this, STZ-diabetic rats treated with APE showed increased levels of GSH, SOD and catalase, thus suggesting the antioxidant capacity of APE.

Andrographis paniculata (AP) possesses antioxidant, antihyperglycemic, antihyperlipidemic and anti-glycation activity and thus, exhibits a protective action in STZinduced diabetic nephropathy in rats. Moreover, further work is necessary to elucidate in detail the mechanism of action of APE at the cellular and molecular levels. 


\section{Acknowledgements}

The authors would express their sincere gratitude to Eureka Research Laboratory staff who assisted the laboratory experiment and

\section{References}

1. Tarigan TJE, Yunir E, Subekti I, Pramono LA, Martina D. Profile and analysis of diabetes chronic complication in outpatient Diabetes Clinic of Cipto Mangunkusumo Hospital, Jakarta. Med J Indo. 2015;24(3):165-70.

2. Papatheodorou K, Banach M, Bekiari E, Rizzo M, Edmonds M. 2018. Complications of diabetes 2017. J Diabetes Res. 2018;3086167.

3. Schena FP, Gesualdo L. Pathogenetic mechanisms of diabetic nephropathy. J Am Soc Nephrol. 2005;Suppl 1:S30-3.

4. Lim AKH. Diabetic nephropathycomplications and treatment. Int J Nephrol Renovasc Dis. 2014;7:361-381.

5. Tanios BY, Ziyadeh FN. Emerging therapies for diabetic nephropathy patients: beyond blockade of the renin-angiotensin system. Nephron Extra. 2012;2:278-282.

6. Ilyas Z, Chaiban JT, Krikorian A. Novel insights into the pathophysiology and clinical aspects of diabetic nephropathy. Rev Endocr Metab Disord. 2017;18(1):21-28.

7. Forbes JM, Coughlan MT, Cooper ME. Oxidative stress as a major culprit in kidney disease in diabetes. Diabetes. 2008;57:1446-54.

8. Darwish NM, Elnahas YM, AlQahtany FS. Diabetes induced renal complications by leukocyte activation of nuclear factor $\kappa-\beta$ and its regulated genes expression. Saudi J Biol Sci. 2020;28(1):541-549.

9. Donousi E, Duni A, Leivaditis K, Vaios V, Eleftheriadis T, Liakopoulos V. Improvements in the management of diabetic nephropathy. Rev Diabet Study. 2015;12(1-2):119-33.

10. Zhang Z, Jiang J, Yu P, Zeng X, Larrick JW, Wang Y. Hypoglycemic and beta cell protective effects of andrographolide analogue for diabetes treatment. J Translat Med. 2009;7:62.

11. Setyaningsih EP, Saputri FC, Mun'im A. The antidiabetic effectivity of Indonesian plants extracts via DPP-IV inhibitory mechanism. J Young Pharmacists. 2019;11(2):161-164. animal care.

The authors declare that there is no conflict of interest.

12. Nugroho AE, Andrie M, Warditiani NK, Siswanto E, Pramono S, Lukitaningsih E. Antidiabetic and antihiperlipidemic effect of Andrographis paniculata (Burm. F.) Nees and andrographolide in high-fructose-fat-fed rats. Indian J Pharmacol. 2012;44(3):377-81.

13. Wediasari F, Nugroho GA, Fadhilah Z, Elya B, Setiawan H, Mozef T. Hypoglicemic effect of a combined Andrographis paniculata and Caesalpinia sappan extract in streptozocininduced diabetic rats. Adv Pharmacol Pharm Sci. 2020.

14. Sari KRP, Sudarsono S, Nugroho AE. Effect of herbal combination of Andrographis paniculata (Burm. F.) Ness and Gynura procumbens (Lour.) Merr ethanolic extracts in alloxan-induced hyperglycemic rats. Int Food Res J. 2015;22(4):1332-1337.

15. Andrade-Cetto A, RubalcabaMares ML. Hypoglycemic effect of the Rhizophora mangle Cortex on STZ-NA induced diabetic rats. Pharmacol online. 2012;3:1-5.

16. King AJ. The use of animal models in diabetes research. Br J Pharmacol. 2012;166(3): 877-94.

17. Nishi AA, Kumar P. Protective effect of chlorogenic acid against diabetic nephropathy in high fat diet/streptozotocin induced type-2 diabetic rats. Int J Pharm Sci. 2013;5:489-95.

18. Halliwell B, Chirico S. Lipid peroxidation: its mechanism, measurement and significance. Am J Clin Nutr. 1993;57(5 Suppl):715S-724S.

19. NIH (National Institute of Health). Principles of laboratory animal care. Bethesda, MD. National Institute of Health, 1985; 1-96.

20. Rocházková D, Bousová I, Wilhelmová N. Antioxidant and prooxidant properties of flavonoids. Fitoterapia. 2011;82(4):513-23.

21. Bastaki S. Diabetes mellitus and its treatment. Int J Diabetes Metab. 2005;13:111-34.

22. Nesbitt KN. An overview of diabetic nephropathy. J Pharm Prac.2004;17(1): 75-9. 
23. Wu KK, Huan Y. Streptozotocin-induced diabetic models in mice and rats. Curr Protoc Pharmacol. 2008; Chapter 5:Unit 5.47.

24. Gheibi S, Kashfi K, Ghasemi A. A practical guide for induction of type-2 diabetes in rat: incorporating a high-fat diet and streptozotocin. Biomed Pharmacother. 2017;95:605-613.

25. Pooranaperundevi M, Sumiyabanu MS, Viswanathan P, Sundarapandiyan R, Anuradha $\mathrm{CV}$. Insulin resistance induced by a high-fructose diet potentiates thioacetamide hepatotoxicity. Singapore Med J. 2010;51(5):389-98.

26. Akhtar MT, Sarib MSM, Ismail IS, Abas F, Ismail A, Lajis $\mathrm{NH}$, et al. Anti-diabetic activity and metabolic changes induced by Andrographis paniculata plant extract in obese diabetic rats. Molecules. 2016;21(8):1026.

27. Zafar M, Naqvi SN. Effects of STZ-induced diabetes on the relative weights of kidney, liver and pancreas in albino rats: a comparative study. Int J Morphol. 2010;28(1):135-142.

28. Viswanathan V, Snehalatha C, Kumutha R, Jayaraman M, Ramachandran A. Serum albumin levels in different stages of type 2 diabetic nephropathy patients. Indian $\mathrm{J}$ Nephrol. 2004;14:89-92.

29. Balbi ME, Tonn FS, Mendes AM, Borba HH, Wiens A, Llimos FF, et al. Antioxidant effects of vitamins in type 2 diabetes: a meta-analysis of randomized controlled trials. Diabetol Metab Syndr. 2018;10:18.

30. Ahmadvand H, Dehnoo MG, Cheraghi R, Rasoulian B, Ezatpour B, Azadpour M, et al. Amelioration of altered serum, liver, and kidney antioxidant enzymes activities by sodium selenite in alloxan-induced diabetic rats. Rep Biochem Mol Bio. 2014;3(1):14-20.

31. Derakhshanian H, Djazayery A, Javanbakht MH, Eshraghian MR, Mirshafiey A, Zarel M, et al. The effect of vitamin D on cellular pathways of diabetic nephropathy. Rep Biochem Mol Bio. 2019;7(2):217-222.

32. Mobasher MA, El-Tantawi HG, El-Said KS. Metformin ameliorates oxidative stress induced by diabetes mellitus and hepatocellular carcinoma in rats. Rep Biochem Mol Bio. 2020;9:115-128. 